\title{
ADMISSIBILITY OF KNEADING SEQUENCES AND STRUCTURE OF HUBBARD TREES FOR QUADRATIC POLYNOMIALS
}

\author{
HENK BRUIN AND DIERK SCHLEICHER
}

\begin{abstract}
Hubbard trees are invariant trees connecting the points of the critical orbits of postcritically finite polynomials. Douady and Hubbard [DH1 introduced these trees and showed that they encode the essential information of Julia sets in a combinatorial way. The itinerary of the critical orbit within the Hubbard tree is encoded by a (pre)periodic sequence on $\{0,1\}$ called kneading sequence.

We prove that the kneading sequence completely encodes the Hubbard tree and its dynamics, and we show how to reconstruct the tree and in particular its branch points (together with their periods, their relative posititions, their number of arms and their local dynamics) in terms of the kneading sequence alone.

Every kneading sequence gives rise to an abstract Hubbard tree, but not every kneading sequence occurs in real dynamics or in complex dynamics. Milnor and Thurston [MT classified which kneading sequences occur in real dynamics; we do the same for complex dynamics in terms of a complex admissibility condition. This complex admissibility condition fails if and only if the abstract Hubbard tree has a so-called evil periodic branch point that is incompatible with local homeomorphic dynamics on the plane.
\end{abstract}

\section{INTRODUCTION}

In complex dynamics, a frequent observation is that many dynamical properties can be encoded in symbolic terms. Douady and Hubbard [DH1] discovered that Julia sets of polynomial Julia sets could completely be described in terms of a tree that is now called the Hubbard tree (at least in the case of postcritically finite polynomials; a complete classification was later given in $[\mathrm{BFH}, \mathrm{Po}])$.

We investigate Hubbard trees of postcritically finite quadratic polynomials. We show that these trees can completely be described by a single periodic binary sequence called kneading sequence which encodes the location of the critical orbit within the tree. More precisely, we show that all endpoints and all branch points of a Hubbard tree are completely encoded by the kneading sequence, and that these suffice to describe the Hubbard tree and its dynamics up to a natural equivalence relation. We show that orbits of branch points come in two kinds which we call tame and evil.

2000 Mathematics Subject Classification. Primary 37F20, Secondary 37B10, 37E25.

Key words and phrases. Hubbard tree, kneading theory, kneading sequence, complex dynamics, Julia set, symbolic dynamics, admissibility condition. 
Kneading sequences are ubiquitous in real and complex dynamics and they have been studied by many people. Milnor and Thurston [MT] classified all kneading sequences that arise in real dynamics, especially by real quadratic polynomials. We answer the corresponding complex question and classify all kneading sequences that arise in complex dynamics: our admissibility condition is given in Definition 4.1. For this, it suffices to restrict attention to sequences that we call $\star$-periodic: it turns out every $\star$-periodic kneading sequence is realized by an essentially unique abstract Hubbard tree; so in order to determine which kneading sequences are realized, we can investigate the associated abstract Hubbard trees. We point out that our trees are abstract in the sense that they do not come with an embedding into the complex plane, in contrast to the original definition of Douady and Hubbard.

Both real and complex admissibility of abstract Hubbard trees are encoded in their branch points: an abstract Hubbard tree is real admissible if it has no branch point at all (the tree is an interval and can be embedded into $\mathbb{R}$ ); an abstract Hubbard tree is complex admissible if it can be embedded into $\mathbb{C}$ so that the embedding respects the circular order. In terms of our classification of branch points, this means that all branch points of the tree must be tame: every evil branch point is an obstruction to complex admissibility of a kneading sequence, and evil branch points are the only possible obstructions. Readers familiar with Thurston's classification [DH2] of rational maps may see similarities with obstructions in that classification. In both cases, a combinatorial obstruction prevents a branched cover, or a Hubbard tree, from being realized by a holomorphic map, in particular by a quadratic polynomial. In fact, our results are closely connected to Thurston's theorem (even though we do not use it).

The simplest example of a non-admissible sequence is $\nu=\overline{10110 \star}$. Here the Hubbard tree, shown in Figure 1, has a period 3 branch point, but the third iterate of $f: T \rightarrow T$ fixes one arm and permutes the other two transitively. Such branch points cannot be embedded into the plane so that the dynamics respects the circular order of the arms: this is an example of an evil branch point.

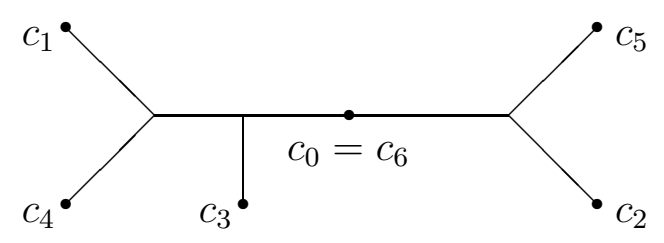

Figure 1 . The Hubbard tree for $1 \rightarrow 2 \rightarrow 4 \rightarrow 5 \rightarrow 6$ contains an evil orbit of period 3 .

The admissibility condition also applies to kneading sequences that are not preperiodic or $\star$-periodic. For the interpretation using evil orbits, finite Hubbard trees have to 
be replaced by dendrites (such as those constructed by Penrose $[\mathrm{Pe}]$ ); another interpetation of complex admissibility is in terms of whether the kneading sequence is realized by angle doubling on the circle. Details, and many further properties of Hubbard trees, are the subject of a forthcoming monograph BKS1.

While Hubbard trees are very good for describing individual Julia sets, it is not quite so easy to tell which trees are close to each other so as to obtain a topology on the space of Hubbard trees. Kneading sequences are helpful here: the natural topology on the space of kneading sequences describes dynamical proximity of Hubbard trees in a way that is compatible, for example, with their location within the Mandelbrot set [BKS1, Section 6].

Kneading sequences can be recoded in "human-readable form" in the form of internal addresses (see Definition 2.4 below): in this form, they allow to read off the location in parameter space of any quadratic polynomial just in terms of the kneading sequence [LS, [S1], and they help to establish fundamental properties of the Mandelbrot set [S2, $\mathrm{HS}]$.

Since all trees in this paper are abstract Hubbard trees, we omit the word "abstract" from now on; one should keep in mind that our definition differs from that by Douady and Hubbard in the fact that their trees always come with an embedding into $\mathbb{C}$. Some of our trees cannot be embedded into the plane in a way that is compatible with the dynamics (those which have evil orbits), while others may have many essentially different such embeddings: such trees are realized by several quadratic polynomials with topologically conjugate dynamics.

The structure of the paper is as follows. In Section 2, we define Hubbard trees and fundamental concepts from symbolic dynamics, including itineraries and kneading sequences. In the rest of the paper, we investigate the Hubbard tree associated to a given $\star$-periodic kneading sequence. Existence and uniqueness of this Hubbard tree are shown in [BKS2]. We do not assume these results here, so the present paper is essentially self-contained: we investigate properties of trees that we assume to exist (but knowing the existence of all these trees reassures us that we are not investigating empty sets). Section 3 contains an investigation of all branch points in Hubbard trees as well as the definition of tame and evil branch points and the proof that the embedding of a tree into the plane respecting the dynamics is possible if and only if all periodic orbits are tame. The final section 4 shows how to determine branch points, their number of arms and their type (evil or tame) in terms of the kneading sequence. We also give a constructive uniqueness proof of Hubbard trees which follows from our investigation of the trees in Corollary 4.20.

Acknowledgement. We gratefully acknowledge that this research was partially supported by the European Marie Curie Research Training Network CODY and by the ESF Research Networking Programme HCAA. HB also acknowledges support by EPSRC grant GR/S91147/01. 


\section{Hubbard Trees}

In this section, we define Hubbard trees as abstract trees with dynamics and show their most fundamental properties. Our trees do not necessarily come with an embedding into the complex plane.

\subsection{Definition (Trees, Arms, Branch Points and Endpoints)}

$A$ tree $T$ is a finite connected graph without loops. For a point $x \in T$, the (global) arms of $x$ are the connected components of $T \backslash\{x\}$. A local arm at $x$ is an intersection of a global arm with a sufficiently small neighborhood of $x$ in $T$. The point $x$ is an endpoint of $T$ if it has only one arm; it is a branch point if it has at least three arms.

Between any two points $x, y$ in a tree, there exists a unique closed arc connecting $x$ and $y$; we denote it by $[x, y]$ and its interior by $(x, y)$.

\subsection{Definition (The Hubbard Tree)}

$A$ Hubbard tree is a tree $T$ equipped with a map $f: T \rightarrow T$ and a distinguished point, the critical point, satisfying the following conditions:

(1) $f: T \rightarrow T$ is continuous and surjective;

(2) every point in $T$ has at most two inverse images under $f$;

(3) at every point other than the critical point, the map $f$ is a local homeomorphism onto its image;

(4) all endpoints of $T$ are on the critical orbit;

(5) the critical point is periodic or preperiodic, but not fixed;

(6) (expansivity) if $x$ and $y$ with $x \neq y$ are branch points or points on the critical orbit, then there is an $n \geq 0$ such that $f^{\circ n}([x, y])$ contains the critical point.

We denote the critical point by $c_{0}=0$ and its orbit by $\operatorname{orb}_{f}\left(c_{0}\right)=\left\{0, c_{1}, c_{2}, \ldots\right\}$. The critical value $c_{1}$ is the image of the critical point. We use a standing assumption that $c_{1} \neq c_{0}$ in order to avoid having to deal with counterexamples when the entire tree is a single point. The branch points and the points on the critical orbit (starting with $c_{0}$ ) will be called marked points. Notice that the set of marked points is finite and forward invariant because the number of arms at any point can decrease under $f$ only at the critical point.

Two Hubbard trees $(T, f)$ and $\left(T^{\prime}, f^{\prime}\right)$ are equivalent if there is a bijection between their marked points which is respected by the dynamics, and if the edges of the tree connect the same marked points. This is weaker than a topological conjugation. In particular, we do not care about details of the dynamics between marked points; there may be intervals of periodic points, attracting periodic points, and so on. (This is related to an equivalence class of branched covers in the sense of Thurston as in [DH2, HS.) 


\subsection{Lemma (The Hubbard Tree)}

The critical value $c_{1}$ is an endpoint, and the critical point $c_{0}$ divides the tree into at most two parts. Each branch point is periodic or preperiodic, it never maps onto the critical point, and the number of arms is constant along the periodic part of its orbit. Any arc which does not contain the critical point in its interior maps homeomorphically onto its image.

Proof. Suppose that $c_{1}$ has at least two arms. The points $c_{2}, c_{3}, \ldots$ also have at least two arms as long as $f$ is a local homeomorphism near this orbit. If this is no longer the case at some point, then the orbit has reached the critical point, and the next image is $c_{1}$ again. In any case, all points on the critical orbit have at least two arms. This contradicts the assumption that all endpoints of a Hubbard tree are on the critical orbit. Hence $c_{1}$ has exactly one arm, and $c_{0}$ has at most two arms (or its image would not be an endpoint).

Since near every non-critical point, the dynamics is a local homeomorphism onto the image, every branch point maps onto a branch point with at least as many arms. Since the critical point has at most two arms, it can never be the image of a branch point. The tree and thus the number of branch points is finite, so every branch point is preperiodic or periodic and its entire orbit consists of branch points; the number of arms is constant along the periodic part of the orbit.

Let $\gamma$ be an arc within the tree. Since $f$ cannot be constant on $\gamma$ and there is no loop in the tree, the subtree $f(\gamma)$ has at least two endpoints. If an endpoint of $f(\gamma)$ is not the image of an endpoint of $\gamma$, then it must be the image of the critical point since $f$ is a local homeomorphism elsewhere, and the critical point 0 must be in the interior of $\gamma$.

In a Hubbard tree $T$ with critical point $c_{0}$, the set $T \backslash\left\{c_{0}\right\}$ consists of at most two connected components; let $T_{1}$ be the component containing the critical value and $T_{0}=T \backslash\left(T_{1} \cup\left\{c_{0}\right\}\right)$ (the set $T_{0}$ may or may not be empty). Writing $T_{\star}=\left\{c_{0}\right\}$, we can define itineraries in the usual way as sequences over $\{0, \star, 1\}$. The itinerary $\nu=\nu_{1} \nu_{2} \nu_{3} \ldots$ of the critical value $c_{1}$ is called the kneading sequence; it always starts with 1. If $c_{0}$ is periodic, say of period $n$, then $\nu_{n}=\star$ and $\nu=\overline{1 \nu_{2} \ldots \nu_{n-1} \star}$; we call such sequences $\star$-periodic.

Write $\mathbb{N}^{*}=\{1,2,3, \ldots\}$ and let $\Sigma^{\star}$ be the set of all $\nu \in\{0,1\}^{\mathbb{N}^{*}}$ and all $\star$-periodic sequences, always subject to the condition that all sequences start with $\nu_{1}=1$.

\subsection{Definition ( $\rho$-Function and Internal Address)}

For a sequence $\nu \in \Sigma^{\star}$, define

$$
\rho_{\nu}: \mathbb{N}^{*} \rightarrow \mathbb{N}^{*} \cup\{\infty\}, \quad \rho_{\nu}(n)=\inf \left\{k>n: \nu_{k} \neq \nu_{k-n}\right\} .
$$

We usually write $\rho$ for $\rho_{\nu}$. For $k \geq 1$, we call

$$
\operatorname{orb}_{\rho}(k):=k \rightarrow \rho(k) \rightarrow \rho^{\circ 2}(k) \rightarrow \rho^{\circ 3}(k) \rightarrow \ldots
$$


the $\rho$-orbit of $k$. The case $k=1$ is the most important one; we call

$$
\operatorname{orb}_{\rho}(1)=1 \rightarrow \rho(1) \rightarrow \rho^{\circ 2}(1) \rightarrow \rho^{\circ 3}(1) \rightarrow \ldots
$$

the internal address of $\nu$. For real unimodal maps, the numbers $\rho^{\circ k}(1)$ are known as the cutting times of the map. If $\rho^{\circ k+1}(1)=\infty$, then we say that the internal address is finite: $1 \rightarrow \rho(1) \rightarrow \ldots \rightarrow \rho^{\circ k}(1)$; as a result, the orbit $\operatorname{orb}_{\rho}$ is a finite or infinite sequence that never contains $\infty$.

The following combinatorial lemma will be used to locate the images of certain closest precritical points in Hubbard trees. The proof can be found in [BKS2, BKS1], and, with entirely different terminology, in the thesis of Penrose [Pe, Theorem 4.5.3 and Corollary 2.5.3.1].

\subsection{Lemma (Combinatorics of $\rho$-Orbits)}

Let $\nu \in \Sigma^{1}$ (not containing $a \star$ ) and let $m$ belong to the internal address of $\nu$.

(1) If $s$ is such that $s<m<\rho(s)$, then $\operatorname{orb}_{\rho}(\rho(m-s)-(m-s)) \ni m$.

(2) If $\rho(m)=\infty$, then $m$ is the exact period of $\nu$.

\section{Periodic Orbits on Hubbard Trees}

In this section, we discuss periodic points of Hubbard trees, in particular branch points, and show that they come in two kinds: tame and evil. This determines whether or not Hubbard trees and kneading sequences are admissible: they are if and only if there is no evil orbit.

\subsection{Lemma (Characteristic Point)}

Let $(T, f)$ be the Hubbard tree with kneading sequence $\nu$. Let $\left\{z_{1}, z_{2}, \ldots, z_{n}=z_{0}\right\}$ be a periodic orbit which contains no endpoint of $T$. If the critical orbit is preperiodic, assume also that the itineraries of all points $z_{k}$ are different from the itineraries of all endpoints of $T$.

Then there are a unique point $z \in\left\{z_{k}\right\}_{k=1}^{n}$ and two different components of $T \backslash\{z\}$ such that the critical value is contained in one component and 0 and all other points $z_{k} \neq z$ are in the other one.

\subsection{Definition (Characteristic Point)}

The point $z$ in the previous lemma is called the characteristic point of the orbit $\left\{z_{k}\right\}$; we will always relabel the orbit cyclically so that the characteristic point is $z_{1}$.

Proof of Lemma 3.1. Note first that every $z_{k} \neq 0$ (or $z_{k+1}=c_{1}$ would be an endpoint). For each $z_{k}$, let $X_{k}$ be the union of all components of $T \backslash\left\{z_{k}\right\}$ which do not contain the critical point. Clearly $X_{k}$ is non-empty and $\left.f\right|_{X_{k}}$ is injective. If $X_{k}$ contains no immediate preimage of 0 , then $f$ maps $X_{k}$ homeomorphically into $X_{k+1}$. Obviously, if $X_{k}$ and $X_{l}$ intersect, then either $X_{k} \subset X_{l}$ or $X_{l} \subset X_{k}$. At least one set $X_{k}$ 
must contain an immediate preimage of 0 : if the critical orbit is periodic, then every endpoint of $T$ eventually iterates onto 0 , and every $X_{k}$ contains an endpoint. If the critical orbit is preperiodic, we need the extra hypothesis on the itinerary of the orbit $\left(z_{k}\right)$ : if no $X_{k}$ contains a point which ever iterates to 0 , then all endpoints of $X_{k}$ have the same itinerary as $z_{k}$ in contradiction to our assumption.

If $X_{k}$ contains an immediate preimage $w$ of 0 , then the corresponding $z_{k}$ separates $w$ from the critical point, i.e., $z_{k} \in[w, 0]$ and thus $z_{k+1} \in\left[0, c_{1}\right]$ (always taking indices modulo $n$ ), hence $c_{1} \in X_{k+1}$.

Among the non-empty set of points $z_{k+1} \in\left[0, c_{1}\right]$, there is a unique one closest to $c_{1}$; relabel the orbit cyclically so that this point is $z_{1}$. We will show that this is the characteristic point of its orbit.

For every $k$, let $n_{k}$ be the number of points from $\left\{z_{i}\right\}$ in $X_{k}$. If $X_{k}$ does not contain an immediate preimage of 0 , then $n_{k+1} \geq n_{k}$. Otherwise, $n_{k+1}$ can be smaller than $n_{k}$, but $z_{k+1} \in\left[0, c_{1}\right]$; since no $z_{k} \in\left(z_{1}, c_{1}\right]$, we have $z_{k+1} \in\left[0, z_{1}\right]$ and either $z_{k+1}=z_{1}$ or $n_{k+1} \geq 1$.

Therefore, if $n_{1} \geq 1$, then all $n_{k} \geq 1$; however, the nesting property of the $X_{k}$ implies that there is at least one 'smallest' $X_{k}$ which contains no further $X_{k^{\prime}}$ and thus no $z_{k^{\prime}}$; it has $n_{k}=0$. Therefore, $n_{1}=0$; this means that all $z_{k} \neq z_{1}$ are in the same component of $T \backslash\left\{z_{1}\right\}$ as 0 . Since $c_{1} \in X_{1}$, the point $z_{1}$ is characteristic.

\subsection{Proposition (Images of Global Arms)}

Let $z_{1}$ be a characteristic periodic point of exact period $m$ and let $G$ be a global arm at $z_{1}$. Then either $0 \notin f^{\circ k}(G)$ for $0 \leq k<m$ (and in particular the first return map of $z_{1}$ maps $G$ homeomorphically onto its image), or the first return map of $z_{1}$ sends the local arm in $G$ to the local arm at $z_{1}$ pointing to the critical point or the critical value.

Proof. Let $z_{k}:=f^{\circ(k-1)}\left(z_{1}\right)$ for $k \geq 1$. Consider the images $f(G), f(f(G))$, etc. of the global arm $G$; if none of them contains 0 before $z_{1}$ returns to itself, then $G$ maps homeomorphically onto its image under the first return map of $z_{1}$ and the claim follows. Otherwise, there is a first index $k$ such that $f^{\circ(k-1)}(G) \ni 0$, so that the image arm at $z_{k}$ points to 0 ; so far, the map is homeomorphic on $G$. If $z_{k}=z_{0}$, then the image point is $z_{1}$ and the local image arm at $z_{1}$ points to $c_{1}$. If $z_{k} \neq z_{0}$, then the local arm at $z_{k}$ points to 0 and the image arm at $z_{k+1}$ points to $c_{1}$; since $z_{1}$ is characteristic, the image arm points also to $z_{1}$. Continuing the iteration, the image arms at the image points will always point to some $z_{l}$. When the orbit finally reaches $z_{0}$, the local arm points to some $z_{l^{\prime}}$. If it also points to 0 , then the image at $z_{1}$ will point to $c_{1}$ as above; otherwise, it maps homeomorphically and the image arm at $z_{1}$ points to $z_{l^{\prime}+1}$. By Lemma 3.1, the only such arm is the arm to the critical point. 


\subsection{Corollary (Two Kinds of Periodic Orbits)}

Let $z_{1}$ be the characteristic point of a periodic orbit of branch points. Then the first return map either permutes all the local arms transitively, or it fixes the arm to 0 and permutes all the other local arms transitively.

Proof. Let $n$ be the exact period of $z_{1}$. Since the periodic orbit does not contain the critical point by Lemma 2.3, the map $f^{\circ n}$ permutes the local arms of $z_{1}$. Let $G$ be any global arm at $z_{1}$. It must eventually map onto the critical point, or the marked point $z_{1}$ would have the same itinerary as all the marked points in $G$, contradicting the expansivity condition. By Lemma 3.3, the orbit of any local arm at $z_{1}$ must include the arm at $z_{1}$ to 0 or to $c_{1}$ or both, and there can be at most two orbits of local arms.

Consider the local arm at $z_{1}$ to 0 . The corresponding global arm cannot map homeomorphically, so $f^{\circ n}$ sends this local arm to the arm pointing to 0 or to $c_{1}$. If the image local arm points to $c_{1}$, then all local arms at $z_{1}$ are on the same orbit, so $f^{\text {on }}$ permutes these arms transitively. If $f^{\circ n}$ fixes the local arm at $z_{1}$ pointing to 0 , then the orbit of every other local arm must include the arm to $c_{1}$, so all the other local arms are permuted transitively.

\subsection{Definition (Tame and Evil Orbits)}

A periodic orbit of branch points is called tame if all its local arms are on the same cycle, and it is called evil otherwise.

REMARK. Obviously, evil orbits are characterized by the property that not all local arms have equal periods; their first return dynamics is described in Corollary 3.4. For periodic points (not containing a critical point) with two local arms, the situation is analogous: the first return map can either interchange these arms or fix them both. It will become clear below that periodic points with only two arms are less interesting than branch points; however, Proposition 3.8 shows that they have similar combinatorial properties.

\subsection{Lemma (Global Arms at Branch Points Map Homeomorphically)}

Let $z_{1}$ be the characteristic point of a periodic orbit of period $n$ and let $q \geq 3$ be the number of arms at each point. Then the global arms at $z_{1}$ can be labelled $G_{0}, G_{1}, \ldots$, $G_{q-1}$ so that $G_{0}$ contains the critical point, $G_{1}$ contains the critical value, and the arms map as follows:

- if the orbit of $z_{1}$ is tame, then the local arm $L_{0} \subset G_{0}$ is mapped to the local arm $L_{1} \subset G_{1}$ under $f^{\circ n}$; the global arms $G_{1}, \ldots, G_{q-2}$ are mapped homeomorphically onto their images in $G_{2}, \ldots, G_{q-1}$, respectively, and the local arm $L_{q-1} \subset G_{q-1}$ is mapped to $L_{0}$;

- if the orbit is evil, then the local arm $L_{0}$ is fixed under $f^{\circ}$, the global arms $G_{1}, \ldots, G_{q-2}$ are mapped homeomorphically onto their images in $G_{2}, \ldots, G_{q-1}$, 
respectively, and the local arm $L_{q-1} \subset G_{q-1}$ is sent to the local arm $L_{1}$; however, the global arm $G_{q-1}$ maps onto the critical point before reaching $G_{1}$.

In particular, if the critical orbit is periodic, then its period must strictly exceed the period of any periodic branch point.

Proof. We will use Proposition 3.3 repeatedly, and we will always use the map $f^{\circ}$. The global arms at $z_{1}$ containing 0 and $c_{1}$ are different because $z_{1} \in\left(0, c_{1}\right)$. If the orbit is tame, then the local arm $L_{0}$ cannot be mapped to itself; since $G_{0} \ni 0, L_{0}$ must map to $L_{1}$. There is a unique local arm at $z_{1}$ which maps to the local arm towards 0 . Let $G_{q-1}$ be the corresponding global arm; it may or may not map onto 0 under $f^{\circ k}$ for $k \leq n$. All the other global arms are mapped onto their images homeomorphically. They can be labelled so that $G_{i}$ maps to $G_{i+1}$ for $i=1,2, \ldots, q-2$. This settles the tame case.

In the evil case, the local arm $L_{0}$ is fixed, and the other local arms are permuted transitively. Let $L_{q-1}$ be the arm for which $f^{\circ n}\left(L_{q-1}\right)$ points to the critical value. Then all other global arms map homeomorphically and can be labelled $G_{1}, G_{2}, \ldots, G_{q-2}$ so that $G_{i}$ maps homeomorphically into $G_{i+1}$ for $i=1,2, \ldots, q-2$.

If $f^{\circ k}\left(G_{q-1}\right) \not \supset 0$ for all $k \leq n$, then the entire cycle $G_{1}, \ldots, G_{q-1}$ of global arms would map homeomorphically onto their images, and all their endpoints would have identical itineraries with $z_{1}$. This contradicts the expansivity condition for Hubbard trees.

\subsection{Corollary (Itinerary of Characteristic Point)}

In the Hubbard tree for the $\star$-periodic kneading sequence $\nu$, fix a periodic point $z$ whose orbit does not contain the critical point. Let $m$ be the period of $z$; if $z$ is not a branch point, suppose that the itinerary of $z$ also has period $m$. Then if the first $m-1$ entries in the itinerary of $z$ are the same as those in $\nu$, the point $z$ is characteristic.

There is a converse if $z$ is a branch point: if $z$ is characteristic, then the first $m$ entries in its itinerary are the same as in $\nu$.

Proof. If $z$ is not characteristic, then by Lemma 3.1, the arc $\left[z, c_{1}\right]$ contains the characteristic point of the orbit of $z$; call it $z^{\prime}$. The itineraries of $z$ and $z^{\prime}$ differ at least once within the period (or the period of the itinerary would divide the period of $z$; for branch points, this would violate the expansivity condition, and otherwise this is part of our assumption). If the itinerary of $z$ coincides with $\nu$ for at least $m-1$ entries, then the same must be true for $z^{\prime} \in\left[z, c_{1}\right]$ (it is easy to check that for any Hubbard tree, the set of points sharing the same $m-1$ entries in their itineraries is connected). Since the number of symbols 0 must be the same in the itineraries of $z$ and $z^{\prime}$, then $z$ and $z^{\prime}$ must have identical itineraries, and this is a contradiction. 
Conversely, if $z$ is the characteristic point of a branch orbit, then by Lemma 3.6, $\left[z, c_{1}\right]$ maps homeomorphically onto its image under $f^{\circ m}$ without hitting 0 , and the first $m$ entries in the itineraries of $z$ and $c_{1}$ coincide.

The following result allows to distinguish tame and evil branch points just by their itineraries.

\subsection{Proposition (Type of Characteristic Point)}

Let $z_{1}$ be a characteristic periodic point. Let $\tau$ be the itinerary of $z_{1}$ and let $n$ be the exact period of $z_{1}$. Then:

- if $n$ occurs in the internal address of $\tau$, then the first return map of $z_{1}$ sends the local arm towards 0 to the local arm toward $c_{1}$, and it permutes all local arms at $z_{1}$ transitively;

- if $n$ does not occur in the internal address of $\tau$, then the first return map of $z_{1}$ fixes the local arm towards 0 and permutes all other local arms at $z_{1}$ transitively.

In particular, a characteristic periodic branch point of period $n$ is evil if and only if the internal address of its itinerary does not contain $n$.

Proof. The idea of the proof is to construct certain precritical points $\zeta_{k_{j}}^{\prime} \in\left[z_{1}, 0\right]$ so that $\left[z_{1}, \zeta_{k_{j}}^{\prime}\right]$ contains no precritical points $\zeta^{\prime}$ with $\operatorname{STEP}\left(\zeta^{\prime}\right) \leq \operatorname{STEP}\left(\zeta_{k_{j}}^{\prime}\right)$. Using these points, the mapping properties of the local arm at $z_{1}$ towards 0 can be investigated. We also need a sequence of auxiliary points $w_{i}$ which are among the two preimages of $z_{1}$.

Let $\zeta_{1}^{\prime}=0$ and $k_{0}=1$ and let $w_{1}$ be the preimage of $z_{1}$ that is contained in $T_{1}$ and let $k_{1} \geq 2$ be maximal such that $\left.f^{\circ\left(k_{1}-1\right)}\right|_{\left[z_{1}, w_{1}\right]}$ is homeomorphic. If $k_{1}<\infty$, then there exists a unique point $\zeta_{k_{1}}^{\prime} \in\left(z_{1}, w_{1}\right)$ such that $f^{\circ k_{1}-1}\left(\zeta_{k_{1}}^{\prime}\right)=0$. All points on $\left[z_{1}, \zeta_{k_{1}}^{\prime}\right)$ have itineraries which coincide for at least $k_{1}-1$ entries. If $k_{1}<n$ then the interval $\left[w_{2}, f^{\circ\left(k_{1}-1\right)}\left(z_{1}\right)\right]$ is non-degenerate and contained in $f^{\circ\left(k_{1}-1\right)}\left(\left(\zeta_{k_{1}}^{\prime}, z_{1}\right]\right)$, where $w_{2}$ denotes the preimage of $z_{1}$ that is not separated from $f^{\circ\left(k_{1}-1\right)}\left(z_{1}\right)$ by 0 . Let $y_{k_{1}} \in\left(\zeta_{k_{1}}^{\prime}, z_{1}\right)$ be such that $f^{\circ\left(k_{1}-1\right)}\left(y_{k_{1}}\right)=w_{2}$. Next, let $k_{2}>k_{1}$ be maximal such that $\left.f^{\circ\left(k_{2}-1\right)}\right|_{\left[z_{1}, y_{k_{1}}\right]}$ is homeomorphic. If $k_{2}<\infty$, then there exists a point $\zeta_{k_{2}}^{\prime} \in\left(z_{1}, \zeta_{k_{1}}^{\prime}\right)$ such that $f^{\circ k_{2}-1}\left(\zeta_{k_{2}}^{\prime}\right)=0$, and the points on $\left[z_{1}, \zeta_{k_{2}}^{\prime}\right)$ have the same itineraries for at least $k_{2}-1$ entries. If $k_{2}<n$ then, as above, the interval $\left[w_{3}, f^{\circ\left(k_{2}-1\right)}\left(z_{1}\right)\right]$ is non-degenerate (where again $w_{3}$ is an appropriate preimage of $\left.z_{1}\right)$ and there is a $y_{k_{2}} \in\left(\zeta_{k_{2}}^{\prime}, z_{1}\right)$ such that $f^{\circ\left(k_{2}-1\right)}\left(y_{k_{2}}\right)=w_{3}$. Continue this way while $k_{j}<n$.

Note that the $\zeta_{k_{j}}^{\prime}$ are among the precritical points on $\left[z_{1}, w_{1}\right]$ closest to $z_{1}$ (in the sense that for each $\zeta_{k_{j}}^{\prime}$, there is no $\zeta^{\prime} \in\left(z_{1}, \zeta_{k_{j}}^{\prime}\right)$ with $\operatorname{STEP}\left(\zeta^{\prime}\right) \leq \operatorname{STEP}\left(\zeta_{k_{j}}^{\prime}\right)$; $\operatorname{compare}$ also Definition 4.5), but the $\zeta_{k_{j}}^{\prime}$ are not all precritical points closest to $z_{1}$; in terms of the cutting time algorithm, the difference can be described as follows: starting with $\left[z_{1}, w_{1}\right]$, we iterate this arc forward until the image contains 0 ; when it does after some 
number $k_{j}$ of iterations, we cut at 0 and keep only the closure of the part containing $f^{\circ k_{j}}\left(z_{1}\right)$ at the end. The usual cutting time algorithm would continue with the entire image arc after cutting, but we cut additionally at the point $w_{j+1} \in f^{-1}\left(z_{1}\right)$.

The point of this construction is the following: let $\rho_{\tau}$ be the $\rho$-function with respect to $\tau$, i.e., $\rho_{\tau}(j):=\min \left\{i>j: \tau_{i} \neq \tau_{i-j}\right\}$. Then $k_{1}=\rho_{\tau}(1)$ and $k_{j+1}=\rho_{\tau}\left(k_{j}\right)$ (if $k \neq 1$, then the exact number of iterations that the arc $\left[z_{1}, z_{k}\right]$ can be iterated forward homeomorphically is $\rho_{\tau}(k)-1$ times). Therefore we have constructed a sequence $\zeta_{k_{j}}^{\prime}$ of precritical points on $\left[z_{1}, 0\right]$ so that (for entries less than $\left.n\right) k_{0}, k_{1}, \cdots=\operatorname{orb}_{\rho_{\tau}}(1)$, which is the internal address associated to $\tau$.

Recall that $n$ is the exact period of $z_{1}$. If $n$ belongs to the internal address, then there exists $\zeta_{n}^{\prime} \in\left[z_{1}, 0\right]$ and $f^{\circ n}$ maps $\left[z_{1}, \zeta_{n}^{\prime}\right]$ homeomorphically onto $\left[z_{1}, c_{1}\right]$. Therefore $f^{\circ n}$ sends the local arm towards 0 to the local arm towards $c_{1}$. By Lemma 3.6, $f^{\circ n}$ permutes all arms at $z_{1}$ transitively.

On the other hand, assume that $n$ does not belong to the internal address. Let $m$ be the last entry in the internal address before $n$. Then $f^{\circ(m-1)} \operatorname{maps}\left[z_{1}, \zeta_{m}^{\prime}\right]$ homeomorphically onto $\left[z_{m}, 0\right]$, and the restriction to $\left[z_{m}, w_{j}\right] \subset\left[z_{m}, 0\right]$ survives another $n-m$ iterations homeomorphically (maximality of $m$ ). There is a point $y_{m} \in\left[z_{1}, 0\right]$ so that $f^{\circ(m-1)}\left(\left[z_{1}, y_{m}\right]\right) \rightarrow\left[z_{m}, w_{j}\right]$ is a homeomorphism, so $f^{\circ n}\left(\left[z_{1}, y_{m}\right]\right) \rightarrow\left[z_{1}, z_{n-m+1}\right]$ is also a homeomorphism. The local arm at $z_{1}$ to 0 maps under $f^{\circ}$ to a local arm at $z_{1}$ to $z_{n-m+1}$, and since $z_{1}$ is characteristic, this means that the local arm at $z_{1}$ to 0 is fixed under the first return map. The other local arms at $z_{1}$ are permuted transitively by Lemma 3.6 .

\subsection{Definition (Admissible Kneading Sequence and Internal Address)}

We call $a \star$-periodic kneading sequence and the corresponding internal address admissible if the associated Hubbard tree contains no evil orbit.

This definition is motivated by the fact that a kneading sequence is admissible if and only if it is realized by a quadratic polynomial; see below.

\subsection{Proposition (Embedding of Hubbard Tree)}

A Hubbard tree $(T, f)$ can be embedded into the plane so that $f$ respects the cyclic order of the local arms at all branch points if and only if $(T, f)$ has no evil orbits.

Proof. If $(T, f)$ has an embedding into the plane so that $f$ respects the cyclic order of local arms at all branch points, then clearly there can be no evil orbit (this uses the fact from Lemma 2.3 that no periodic orbit of branch points contains a critical point).

Conversely, suppose that $(T, f)$ has no evil orbits, so all local arms at every periodic branch point are permuted transitively. First we embed the arc $\left[0, c_{1}\right]$ into the plane, for example on a straight line. Every cycle of branch points has at least its characteristic point $p_{1}$ on the arc $\left[0, c_{1}\right]$, and it does not contain the critical point. Suppose $p_{1}$ has $q$ arms. Take $s \in\{1, \ldots, q-1\}$ coprime to $q$ and embed the local arms at $p_{1}$ in such 
a way that the return map $f^{\circ n}$ moves each arc over by $s$ arms in counterclockwise direction. This gives a single cycle for every $s<q$ coprime to $q$. Furthermore, this can be done for all characteristic branch points independently.

We say that two marked points $x, y$ are adjacent if $(x, y)$ contains no further marked point. If a branch point $x$ is already embedded together with all its local arms, and $y$ is an adjacent marked point on $T$ which is not yet embedded but $f(y)$ is, then draw a line segment representing $[x, y]$ into the plane, starting at $x$ and disjoint from the tree drawn so far. This is possible uniquely up to homotopy. Embed the local arms at $y$ so that $f: y \rightarrow f(y)$ respects the cyclic order of the local arms at $y$; this is possible because $y$ is not the critical point of $f$.

Applying the previous step finitely many times, the entire tree $T$ can be embedded. It remains to check that for every characteristic branch point $p_{1}$ of period $m$, say, the map $f: p_{1} \rightarrow f\left(p_{1}\right)=: p_{2}$ respects the cyclic order of the local arms. By construction, the forward orbit of $p_{2}$ up to its characteristic point $p_{1}$ is embedded before embedding $p_{2}$, and $f^{\circ(m-1)}: p_{2} \rightarrow p_{1}$ respects the cyclic order of the embedding. If the orbit of $p_{1}$ is tame, the cyclic order induced by $f: p_{1} \rightarrow p_{2}$ (from the abstract tree) is the same as the one induced by $f^{\circ(m-1)}: p_{2} \rightarrow p_{1}$ used in the construction (already embedded in the plane), and the embedding is indeed possible.

REMARK. It is well known that once the embedding respects the cyclic order of the local arms and their dynamics, then the map $f$ extends continuously to a neighborhood of $T$ within the plane, and even to a branched cover of the sphere with degree 2 . See for example [BFH]. This implies that the kneading sequence of $T$ is generated by an external angle and that $T$ occurs as the Hubbard tree of a quadratic polynomial (compare [BKS1]).

It is not difficult to determine the number of different embeddings of $T$ into the plane (where we consider two embeddings of a Hubbard tree into the plane as equal if the cyclic order of all the arms at each branch point is the same): if $q_{1}, q_{2}, \ldots, q_{k}$ are the number of arms at the different characteristic branch points and $\varphi(q)$ is the Euler function counting the positive integers in $\{1,2, \ldots, q-1\}$ that are coprime to $q$, then the number of different embeddings of $T$ respecting the dynamics is $\prod_{i} \varphi\left(q_{i}\right)$ [BKS1, Section 5]. This also counts the number of times $T$ is realized as the Hubbard tree of a postcritically finite quadratic polynomial. If the critical orbit is periodic of period $n$, then it turns out that $\prod_{i} \varphi\left(q_{i}\right)<n$; see [BKS1, Section 16].

\section{The Admissibility Condition}

In this section we derive the nature of periodic branch points of the Hubbard tree from the kneading sequence (Propositions 4.13 and 4.19). We also prove a condition (admissibility condition) on the kneading sequence which decides whether there are evil orbits: Proposition 4.12 shows that an evil orbit violates this condition, and Proposition 4.13 shows that a violated condition leads to an evil orbit within the Hubbard tree. Since a Hubbard tree 
can be embedded in the plane whenever there is no evil orbit (Proposition 3.10), we obtain a complete classification of admissible kneading sequences (Theorem 4.2).

\subsection{Definition (The Admissibility Condition)}

A kneading sequence $\nu \in \Sigma^{\star}$ fails the admissibility condition for period $m$ if the following three conditions hold:

(1) the internal address of $\nu$ does not contain $m$;

(2) if $k<m$ divides $m$, then $\rho(k) \leq m$;

(3) $\rho(m)<\infty$ and if $r \in\{1, \ldots, m\}$ is congruent to $\rho(m)$ modulo $m$, then $\operatorname{orb}_{\rho}(r)$ contains $m$.

A kneading sequence fails the admissibility condition if it does so for some $m \geq 1$.

An internal address fails the admissibility condition if its associated kneading sequence does.

This definition applies to all sequences in $\Sigma^{\star}$, i.e., all sequences in $\{0,1\}^{\mathbb{N}^{*}}$ and all $\star$-periodic sequences, provided they start with 1 . However, in this section and the next we will only consider $\star$-periodic and preperiodic kneading sequences because these are the ones for which we have Hubbard trees. The main result in this section is that this condition precisely describes admissible kneading sequences in the sense of Definition 3.9 (those for which the Hubbard tree has no evil orbits):

\subsection{Theorem (Evil Orbits and Admissibility Condition)}

A Hubbard tree contains an evil orbit of exact period $m$ if and only if its kneading sequence fails the Admissibility Condition 4.1 for period $m$.

Equivalently, a Hubbard tree can be embedded into the plane so that the dynamics respects the embedding if and only if the associated kneading sequence does not fail the Admissibility Condition 4.1 for any period.

The proof of the first claim will be given in Propositions 4.12 and 4.13 , and the second is equivalent by Proposition 3.10 .

\subsection{Example (Non-Admissible Kneading Sequences)}

The internal address $1 \rightarrow 2 \rightarrow 4 \rightarrow 5 \rightarrow 6$ with kneading sequence $\overline{10110 \star}$ (or any address that starts with $1 \rightarrow 2 \rightarrow 4 \rightarrow 5 \rightarrow 6 \rightarrow$ ) fails the admissibility condition for $m=3$, and the Hubbard tree indeed has a periodic branch point of period 3 that does not permute its arms transitively, as can be verified in Figure 1. This is the simplest and best known example of a non-admissible Hubbard tree; see [LS, $\mathrm{Ke}, \mathrm{Pe}$.

More generally, let $\nu=\overline{\nu_{1} \ldots \nu_{m-1}}$ be any $\star$-periodic kneading sequence of period $m$ so that there is no $k$ dividing $m$ with $\rho(k)=m$. This clearly implies $\rho(k)<m$ for all $k$ dividing $m$. Let $\nu_{m} \in\{0,1\}$ be such that $m$ does not occur in the internal address of $\overline{\nu_{1} \ldots \nu_{m}}$. Then for any $s \geq 2$, every sequence starting with

$$
\overline{\underbrace{\nu_{1} \ldots \nu_{m} \ldots \nu_{1} \ldots \nu_{m}}_{s-1 \text { times }} \nu_{1} \ldots \nu_{m-1} \nu_{m}^{\prime}}
$$


(with $\nu_{m}^{\prime} \neq \nu_{m}$ ) fails the admissibility condition for $m$. The example $1 \rightarrow 2 \rightarrow 4 \rightarrow$ $5 \rightarrow 6$ above with kneading sequence $\overline{10110 \star}$ has been constructed in this way, starting from $\overline{10 \star}$.

It is shown in $[\mathrm{K}$ that every non-admissible kneading sequence is related to such an example: the kneading sequences as constructed in this example are exactly those where within the tree of admissible kneading sequences, subtrees of non-admissible sequences branch off (compare also [BKS1, Section 6]). These sequences correspond exactly to primitive hyperbolic components of the Mandelbrot set.

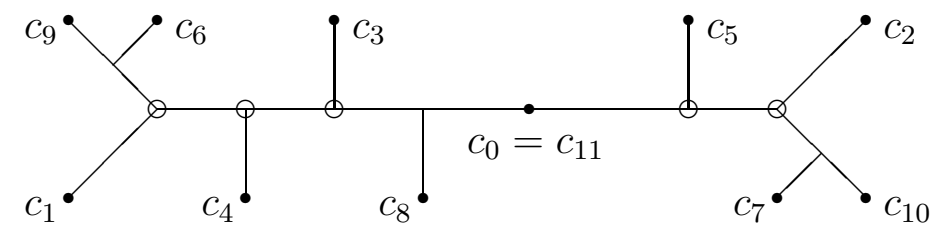

FiguRE 2 . The Hubbard tree for $1 \rightarrow 2 \rightarrow 4 \rightarrow 5 \rightarrow 11$ is admissible. There is a tame periodic orbit of branch points of period 5 (indicated by o's). The other branch points are preperiodic.

While $1 \rightarrow 2 \rightarrow 4 \rightarrow 5 \rightarrow 6$ is not admissible, the internal address $1 \rightarrow 2 \rightarrow 4 \rightarrow 5 \rightarrow$ 11 is admissible; its Hubbard tree is shown in Figure 2. This shows that the Translation Principle from [LS, Conjecture 8.7] does not hold: the address $1 \rightarrow 2 \rightarrow 4 \rightarrow 5 \rightarrow 11$ is realized in the $\frac{1}{3}$ and $\frac{2}{3}$-sublimbs of the real period 5 component $1 \rightarrow 2 \rightarrow 4 \rightarrow 5$ of the Mandelbrot set. The Translation Principle would predict that $1 \rightarrow 2 \rightarrow 4 \rightarrow 5 \rightarrow 6$ should exist within the $\frac{1}{2}$-sublimb, but no such hyperbolic component exists (the same counterexample was found independently by V. Kauko [Ka]).

REMARK. The three conditions in the admissibility condition are independent: here are examples of kneading sequences where exactly two of the three conditions are satisfied.

- $\nu=\overline{101 \star}(1 \rightarrow 2 \rightarrow 4), m=2$ : condition 1 is violated; $\nu$ is admissible.

- $\nu=\overline{111 \star}(1 \rightarrow 4), m=2$ : condition 2 is violated; $\nu$ is admissible.

- $\nu=\overline{101 \star}(1 \rightarrow 2 \rightarrow 4), m=3$ : condition 3 is violated; $\nu$ is admissible.

These conditions can be interpreted as follows: the first condition picks a candidate period for an evil orbit, taking into account that a branch point is always tame when its period occurs on the internal address (Proposition 4.12); the second condition assures that the period $m$ of the evil orbit is the exact period, and the third condition makes the periodic orbit evil by assuring that the first return map of the characteristic point maps a different local arm than the one pointing to 0 onto the local arm to the critical value. 


\subsection{Lemma (Bound on Failing the Admissibility Condition)}

If a -periodic kneading sequence of period $n$ fails the admissibility condition for period $m$, then $m<n$.

Proof. Since $n$ occurs in the internal address, we may suppose $m \neq n$. If $m>n$, then $\rho(m)<m+n$ (because one of the entries between $m$ and $m+n$ is a $\star$ ), hence $r<n$ and $\operatorname{orb}_{\rho}(r)$ terminates at $n$, so $m \notin \operatorname{orb}_{\rho}(r)$.

A different way to interpret Lemma 4.4 is to say that a $\star$-periodic kneading sequence fails the admissibility condition for period $m$ if and only if the associated Hubbard tree has an evil branch point of period $m$ (Theorem 4.2), and the period of a branch point is bounded by the period of the kneading sequence (Lemma 3.6).

One of the main tools are closest precritical points.

\subsection{Definition (Precritical Points)}

A point $x \in T$ is called precritical if $f^{\circ k}(x)=c_{1}$ for some $k \geq 1$; the least such index $k$ is called $\operatorname{STEP}(x)$. The point $x$ is called a closest precritical point and denoted $\zeta_{k}$ if $f^{\circ j}\left(\left[c_{1}, x\right]\right) \not \supset c_{1}$ for all $j \in\{1, \ldots, k-1\}$.

The critical point is always $\zeta_{1}$; if the critical point is periodic of period $n$, then $\zeta_{n}=c_{1}$ and there is no closest precritical point $x$ with $\operatorname{STEP}(x)>n$. Closest precritical points are those which are "visible from $c_{1}$ " in the sense of [LS, Section 8]: the idea is that a precritical point $\zeta$ blocks the view of all $\zeta^{\prime}$ behind $\zeta$ with $\operatorname{STEP}\left(\zeta^{\prime}\right) \geq \operatorname{STEP}(\zeta)$ (figuratively speaking, $\zeta$ is so big that the smaller point $\zeta^{\prime}$ cannot be seen if it is behind $\zeta)$. We say that $\zeta$ is the earliest precritical point on an $\operatorname{arc}(x, y)$ (or $[x, y]$ etc.) if it is the one with the lowest STEP.

\subsection{Lemma (Closest Precritical Points Unique)}

A Hubbard tree contains at most one closest precritical point $\zeta_{k}$ for every index $k$.

Proof. If for some $k$, there are two closest precritical points $\zeta_{k}$ and $\zeta_{k}^{\prime}$, then $f^{\circ(k-1)}$ maps $\left[\zeta_{k}, \zeta_{k}^{\prime}\right]$ homeomorphically onto its image, but both endpoints map to the critical point 0 . This is a contradiction.

\subsection{Lemma (Elementary Properties of $\rho$ )}

If $\zeta_{k} \neq c_{1}$, then the earliest precritical point on $\left(\zeta_{k}, c_{1}\right]$ is $\zeta_{\rho(k)}$. For $k \geq 1$, the earliest precritical point on $\left[c_{1+k}, c_{1}\right)$ is $\zeta_{\rho(k)-k}$.

If $\zeta_{k} \neq c_{1}$, then $\left[c_{1}, \zeta_{k}\right]$ contains those and only those closest precritical points $\zeta_{m}$ for which $m \in \operatorname{orb}_{\rho}(k)$. In particular, $\zeta_{m} \in\left[0, c_{1}\right]$ if and only if $m$ belongs to the internal address.

Proof. The first two statements follow immediately from the definition of $\rho$, using the idea of cutting times, namely that if we look at the largest neighborhood of $c_{1}$ in $T$ 
on which $f^{\circ k}$ is monotone, we have cut this neighborhood at a closest precritical point $\zeta_{n}$ whenever $n=k-1$. Note that the arc $\left[c_{1}, \zeta_{k}\right)$ can be iterated homeomorphically for at least $k$ iterations, and $f^{\circ k}\left(\left[c_{1}, \zeta_{k}\right)\right)=\left[c_{1+k}, c_{1}\right)$. The first time that $f^{\circ(m-1)}\left(\left[c_{1}, \zeta_{k}\right)\right)$ hits 0 is for $m=\rho(k)$ by definition, and the earliest precritical point on $\left[c_{1}, \zeta_{k}\right)$ takes exactly $\rho(k)$ steps to map to $c_{1}$. The claim now follows by induction. The statement about the internal address follows because $\zeta_{1}=0$.

\subsection{Lemma (Images of Closest Precritical Points) \\ If $k<k^{\prime} \leq \rho(k)$, then $f^{\circ k}\left(\zeta_{k^{\prime}}\right)$ is the closest precritical point $\zeta_{k^{\prime}-k}$.}

Proof. Let $x:=f^{\circ k}\left(\zeta_{k^{\prime}}\right)$. Then the arc $\left[c_{1}, \zeta_{k^{\prime}}\right]$ maps under $f^{\circ k}$ homeomorphically onto $\left[c_{k+1}, x\right]$, and there is no precritical point $\zeta \in\left(c_{k+1}, x\right)$ with $\operatorname{STEP}(\zeta) \leq k^{\prime}-k$. If $\rho(k)>k^{\prime}$ then by Lemma 4.7 there is no such precritical point $\zeta \in\left[c_{1}, c_{k+1}\right]$ either, and hence none on $\left(x, c_{1}\right]$. Since $\operatorname{SteP}(x)=k^{\prime}-k$, the point $x$ is indeed the closest precritical point $\zeta_{k^{\prime}-k}$. Finally, if $\rho(k)=k^{\prime}$, then $\zeta_{\rho(k)-k}=\zeta_{k^{\prime}-k}$ is the earliest precritical point on $\left[c_{1}, c_{1+k}\right]$ (Lemma 4.7). But since $x$ also has $\operatorname{STEP}(x)=k^{\prime}-k$ and $\left[x, \zeta_{k^{\prime}-k}\right]$ contains no point of lower STEP, we have $x=\zeta_{k^{\prime}-k}$.

\subsection{Lemma (Precritical Points Near Periodic Points)}

Let $z_{1}$ be a characteristic periodic point of period $m$ such that $f^{\circ m}$ maps $\left[z_{1}, c_{1}\right]$ homeomorphically onto its image. Assume that $\nu$ is not $\star$-periodic of period less than $m$. If $z_{1}$ has exactly two local arms, assume also that the first return map of $z_{1}$ interchanges them. Then

(1) the closest precritical point $\zeta_{m}$ exists in the Hubbard tree, $z_{1} \in\left[\zeta_{m}, c_{1}\right]$ and $\zeta_{\rho(m)} \in\left[c_{1}, z_{1}\right]$

(2) if $\zeta$ is a precritical point closest to $z_{1}$ with $\operatorname{STEP}(\zeta)<m$ in the same global arm of $z_{1}$ as $\zeta_{m}$, then $\zeta_{m} \in\left[z_{1}, \zeta\right]$;

(3) if $z_{1}$ is a tame branch point, then $\zeta_{m} \in\left[0, z_{1}\right]$ and $m$ occurs in the internal address;

(4) if $z_{1}$ is an evil branch point, then $\zeta_{m} \in G_{q-1}$ (where global arms are labelled as in Lemma 3.6) and $m$ does not occur in the internal address.

Proof. (1) First we prove the existence of $\zeta_{m}$ in $T$. Let $G_{0}, G_{1}, \ldots, G_{q-1}$ be the global arms of $z_{1}$ with $0 \in G_{0}$ and $c_{1} \in G_{1}$. (Note that $q=2$ is possible.) Let $L_{0}, \ldots, L_{q-1}$ be the corresponding local arms. Let $j$ be such that $f^{\circ m}\left(L_{j}\right)=L_{1}$.

If $j=0$, then $0=\zeta_{1} \in G_{j}$. If $j \neq 0$, then $q \geq 3$ by assumption, so $j=q-1$ by Lemma 3.6 and there is an $i<m$ so that $f^{\circ i}\left(G_{j}\right)$ contains 0 . Therefore, in both cases there exists a unique $\zeta_{k} \in G_{j}$ with $k \leq m$ maximal, and it satisfies $z_{1} \in\left(\zeta_{k}, c_{1}\right)$. We want to show that $k=m$.

If $k<m$, then $f^{\circ k}$ maps $\left(z_{1}, \zeta_{k}\right)$ homeomorphically onto $\left(z_{k+1}, c_{1}\right) \ni z_{1}$. By maximality of $k$, the restriction of $f^{\circ m}$ to $\left(z_{1}, \zeta_{k}\right)$ is a homeomorphism with image 
$\left(z_{1}, c_{m-k+1}\right) \subset G_{1}$, and it must contain $f^{\circ(m-k)}\left(z_{1}\right)=z_{1+m-k}$ in contradiction to the fact that $z_{1}$ is characteristic. Hence $k=m, \zeta_{m}$ exists and $z_{1} \in\left[c_{1}, \zeta_{m}\right]$.

Clearly $f^{\circ m}$ maps $\left[z_{1}, \zeta_{m}\right]$ homeomorphically onto $\left[z_{1}, c_{1}\right]$. By Lemma $4.7, \zeta_{\rho(m)} \in$ $\left[c_{1}, \zeta_{m}\right]$. If $\zeta_{\rho(m)} \in\left[z_{1}, \zeta_{m}\right]$, then $f^{\circ m}\left(\zeta_{\rho(m)}\right) \in\left[c_{1}, z_{1}\right] \subset\left[c_{1}, \zeta_{\rho(m)}\right]$, but then $\zeta_{\rho(m)}$ would not be a closest precritical point. Hence $\zeta_{\rho(m)} \in\left[z_{1}, c_{1}\right]$.

(2) For the second statement, let $k:=\operatorname{STEP}(\zeta)<m$. We may suppose that $\left(z_{1}, \zeta\right)$ contains no precritical point $\zeta^{\prime \prime}$ with $\operatorname{STEP}\left(\zeta^{\prime \prime}\right)<m$ (otherwise replace $\zeta$ by $\zeta^{\prime \prime}$ ). Clearly $\zeta \notin\left[z_{1}, \zeta_{m}\right]$. Assume by contradiction that $\zeta_{m} \notin\left[z_{1}, \zeta\right]$ so that $\left[z_{1}, \zeta, \zeta_{m}\right]$ is a nondegenerate triod. Since both $\left[z_{1}, \zeta\right]$ and $\left[z_{1}, \zeta_{m}\right]$ map homeomorphically under $f^{\circ m}$, the same is true for the triod $\left[z_{1}, \zeta, \zeta_{m}\right]$.

Under $f^{\circ k}$, the triod $\left[z_{1}, \zeta, \zeta_{m}\right]$ maps homeomorphically onto the triod $\left[z_{k+1}, c_{1}, \zeta^{\prime}\right]$ with $\operatorname{STEP}\left(\zeta^{\prime}\right)=m-k$, and $z_{k+1} \neq z_{1}$. Then $z_{1} \in\left(z_{k+1}, c_{1}\right)$, so the arc $\left(z_{k+1}, \zeta^{\prime}\right)$ contains either $z_{1}$ or a point at which the path to $z_{1}$ branches off. Under $f^{\circ(m-k)}$, the triod $\left[z_{k+1}, c_{1}, \zeta^{\prime}\right] \ni z_{1}$ maps homeomorphically onto $\left[z_{1}, c_{m-k+1}, c_{1}\right] \ni z_{m-k+1}$. Therefore $\left(z_{1}, c_{1}\right)$ contains either the point $z_{m-k+1}$ or a branch point from which the path to $z_{m-k+1}$ branches off. Both are in contradiction to the characteristic property of $z_{1}$.

(3) If $z_{1}$ is tame, then $j=0$, so $\zeta_{m} \in G_{0}$. By the previous statement, $\zeta_{m} \in\left[z_{1}, 0\right]$, and Lemma 4.7 implies that $m$ belongs to the internal address.

(4) Finally, if $z_{1}$ is evil, then $\zeta_{m} \in G_{q-1}$ and $m$ does not occur in the internal address by Lemma 4.7 .

The following lemma is rather trivial, but helpful to refer to in longer arguments.

\subsection{Lemma (Translation Property of $\rho$ )}

If $\rho(m)>k m$ for $k \geq 2$, then $\rho(k m)=\rho(m)$.

Proof. Let $\nu$ be a kneading sequence associated to $\rho$. Then $\rho(m)>k m$ says that the first $m$ entries in $\nu$ repeat at least $k$ times, and $\rho(m)$ finds the first position where this pattern is broken. By definition, $\rho(\mathrm{km})$ does the same, omitting the first $k$ periods.

\subsection{Lemma (Bound on Number of Arms)}

Let $z_{1} \in\left[c_{1}, \zeta_{m}\right]$ be a characteristic point of period $m$ with $q$ arms. Assume that $\nu$ is not *-periodic of period less than $m$. If $z_{1}$ has exactly two local arms, assume also that the first return map of $z_{1}$ interchanges them. If $z_{1}$ is evil, then $(q-2) m<\rho(m) \leq(q-1) m$; if not, then $(q-2) m<\rho(m) \leq q m$.

Proof. Let $G_{0} \ni 0, G_{1} \ni c_{1}, \ldots, G_{q-1}$ be the global arms at $z_{1}$. By Lemma 4.9, $\zeta_{\rho(m)} \in\left[z_{1}, c_{1}\right]$. The lower bound for $\rho(m)$ follows from Lemma 3.6.

First assume that $z_{1}$ is evil, so $q \geq 3$. Lemma 4.10 implies $\rho((q-2) m)=\rho(m)$. Assume by contradiction that $\rho(m)>(q-1) m$. Then $r:=\rho(m)-(q-2) m>$ $m$. By Lemma 4.8, $\zeta_{r}=f^{\circ((q-2) m)}\left(\zeta_{\rho(m)}\right)$ is a closest precritical point. It belongs to the same arm $G_{q-1}$ as $\zeta_{m}$, but $\zeta_{m} \notin\left[z_{1}, \zeta_{r}\right]$. As $\zeta_{\rho(m)}$ is the earliest precritical 
point on $\left[c_{1}, \zeta_{m}\right)$, we cannot have $\zeta_{r} \in\left[z_{1}, \zeta_{m}\right]$ either. Therefore $\left[z_{1}, \zeta_{r}, \zeta_{m}\right]$ is a nondegenerate triod within $\bar{G}_{q-1}$; let $y \in G_{q-1}$ be the branch point, see Figure 3 . Obviously

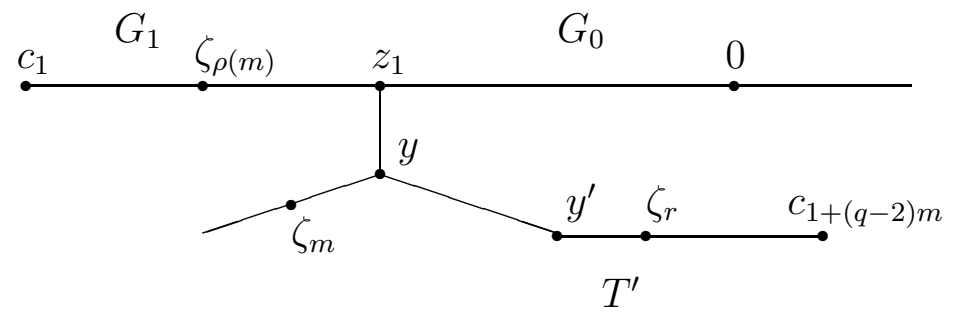

FiguRE 3. Subtree with an evil branch point $z_{1}$ of a Hubbard tree.

$f^{\circ m}(y) \in\left[z_{1}, c_{1}\right]$ and since $f^{\circ((q-2) m)}$ maps $G_{1}$ homeomorphically into $G_{q-1}$, we find $y^{\prime}:=f^{\circ((q-1) m)}(y) \in\left[z_{1}, c_{1+(q-2) m}\right]$, see Figure 3. If $y^{\prime} \in\left[z_{1}, y\right]$, then $f^{\circ((q-1) m}$ maps $\left[z_{1}, y\right]$ homeomorphically into itself. This contradicts expansivity of the tree. Therefore $y^{\prime} \in\left(y, c_{1+(q-2) m}\right]$. Let $T^{\prime}$ be the component of $T \backslash\{y\}$ containing $c_{1+(q-2) m}$. Since $\zeta_{\rho(m)} \in\left[z_{1}, c_{1}\right]$ and $f^{\circ(q-2) m}\left(\zeta_{\rho(m)}\right)=\zeta_{r}, T^{\prime}$ contains $\zeta_{r}$ but not $\zeta_{m}$. Now $f^{\circ((q-1) m)}$ maps $T^{\prime}$ homeomorphically into itself (otherwise, there would be an earliest precritical point $\zeta \in T^{\prime}$ with $\operatorname{STEP}(\zeta)<m$, but then $\zeta_{m} \in\left[z_{1}, \zeta\right]$ by Lemma 4.9 (2)). Again, expansivity of the tree is violated. Thus indeed $\rho(m) \leq(q-1) m$.

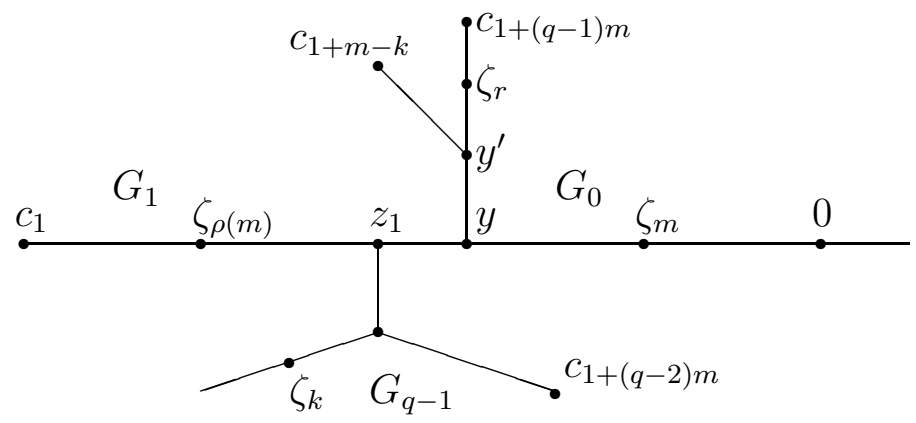

FiguRE 4 . Subtree with a tame branch point $z_{1}$ of a Hubbard tree.

Now assume that $z_{1}$ is not evil (and maybe not even a branch point). Assume by contradiction that $\rho(m)>q m$. We repeat the above argument with $r:=\rho(m)-(q-$ 1) $m>m$, conclude that $\rho((q-1) m)=\rho(m)$ and find the closest precritical point $\zeta_{r} \in G_{0}$, so $\zeta_{r}$ is in the same global arm at $z_{1}$ as $\zeta_{m}$. As before, $\left[z_{1}, \zeta_{r}, \zeta_{m}\right]$ is a nondegenerate triod with branch point $y$ and $y^{\prime}:=f^{\circ q m}(y)$ lies on $\left[y, c_{1+(q-1) m}\right]$. Let $T^{\prime}$ be 
the component of $T \backslash\{y\}$ containing $c_{1+(q-1) m}$. We claim that $f^{\circ q m}$ is homeomorphic on $T^{\prime}$.

It follows as above, using Lemma 4.9, that $f^{\circ m}$ maps $T^{\prime}$ homeomorphically into $G_{1}$, and $f^{\circ(q-2) m}$ maps $G_{1}$ homeomorphically into $G_{q-1}$. Let $T^{\prime \prime}=f^{\circ(q-1) m}\left(T^{\prime}\right)$ and assume by contradiction that $f^{\circ m}$ is not homeomorphic on $T^{\prime \prime}$. Then $T^{\prime \prime}$ contains a closest precritical point $\zeta_{k}$ for some $k<m$. Take $k<m$ maximal. Then $f^{\circ m}$ is homeomorphic on $\left[z_{1}, \zeta_{k}\right]$, and since $f^{\circ k}\left(\left[z_{1}, \zeta_{k}\right]\right)=\left[z_{k+1}, c_{1}\right] \ni z_{1}$, it follows that $f^{\circ m}\left(\left[z_{1}, \zeta_{k}\right]\right)=\left[z_{1}, c_{1+m-k}\right]$ contains $z_{1+m-k}$. But since $\zeta_{k} \in T^{\prime \prime}$, hence $f^{\circ(q-1) m}(y) \in$ $\left[z_{1}, \zeta_{k}\right]$, we also have $y^{\prime} \in\left[z_{1}, c_{1+m-k}\right]$. As a result, $\left[z_{1}, c_{1+m-k}\right] \subset\left[z_{1}, y\right] \cup T^{\prime}$.

If $z_{1+m-k} \in\left[z_{1}, y\right]$, then $f^{\circ m}$ maps $\left[z_{1}, z_{1+m-k}\right]$ homeomorphically onto its image. This is a contradiction: both endpoints are fixed, but the image must be in $G_{1}$. Therefore, $z_{1+m-k} \in T^{\prime}$. By Lemma 4.9 (2) again, there can be no precritical point with STEP less than $m$ on $\left[z_{1}, z_{1+m-k}\right]$, and we get the same contradiction.

We can conclude as above that $f^{\circ q m}$ maps $T^{\prime}$ homeomorphically into itself as claimed. But this is a contradiction to expansivity of the tree.

\subsection{Proposition (Evil Orbit Fails Admissibility Condition)}

If a Hubbard tree has an evil orbit of exact period $m$ and $\nu$ is not $\star$-periodic of period less than $m$, then the kneading sequence fails the admissibility condition for period $m$.

Proof. Let $z_{1}$ be the characteristic point of the evil orbit of period $m$ and let $G_{0}, \ldots, G_{q-1}$ be the global arms labelled as in Lemma 3.6. The corresponding local arms will be labelled $L_{0}, \ldots, L_{q-1}$.

We know from Lemma 4.9 that $m$ is not in the internal address, so the first part of the admissibility condition is already taken care of. Since $\left[z_{1}, c_{1}\right]$ maps homeomorphically for $m$ steps, the first $m$ entries in the itineraries of $z_{1}$ and $c_{1}$ coincide, and $e\left(z_{1}\right)=$ $\overline{\nu_{1} \ldots \nu_{m}}$. Let $k<m$ be a divisor of $m$. Suppose by contradiction that $\rho(k)>m$. Then $e\left(z_{1}\right)$ has period $k$.

Therefore the period of $z_{1}$ is a multiple, and if it is a proper multiple, then $z_{1}$ and $f^{\circ k}\left(z_{1}\right)$ are two periodic points with the same itinerary. This contradicts expansivity of the Hubbard tree, so the period of $z_{1}$ must be $k$ as well. This however contradicts the assumption, settling the second condition of Definition 4.1

Let $r:=\rho(m)-(q-2) m$. By Lemma 4.11, $0<r \leq m$. By Lemma 4.8, $f^{\circ(q-2) m}\left(\zeta_{\rho(m)}\right)=\zeta_{\rho(m)-(q-2) m}=\zeta_{r} \in G_{q-1}$.

By Lemma4.9 (4) and (2), we have $\zeta_{m} \in G_{q-1}$ and then $\zeta_{m} \in\left[z_{1}, \zeta_{r}\right]$. Now Lemma4.7 shows that $m \in \operatorname{orb}_{\rho}(r)$. Hence $\nu$ fails the admissibility condition for period $m$.

In Propositions 4.13 and 4.19, we will determine the exact number of arms at all branch points, and determine from the internal address which branch points a Hubbard tree has. 


\subsection{Proposition (Number of Arms at Evil Branch Points)}

Suppose a kneading sequence $\nu$ fails the Admissibility Condition 4.1 for period $m$, and that $\nu$ is not $\star$-periodic of period less than $m$. Then the Hubbard tree for $\nu$ contains an evil branch point of exact period $m$; the number of its arms is $q:=\lfloor\rho(m) / m\rfloor+2 \geq 3$.

Proof. Write $\rho(m)=(q-2) m+r$ for $r \in\{1,2, \ldots, m\}$ and $q \geq 3$. Then $\rho((q-$ 2) $m)=\rho(m)$ by Lemma 4.10 and the earliest precritical point on $\left[c_{1+(q-2) m}, c_{1}\right)$ is $\zeta_{r}$ by Lemma 4.7. Since $\nu$ fails the admissibility condition for $m$, this implies in particular $m \in \operatorname{orb}_{\rho}(r)$, hence by Lemma $4.7 \zeta_{m} \in\left[\zeta_{r}, c_{1}\right] \subset\left[c_{1+(q-2) m}, c_{1}\right]$.

Consider the connected hull

$$
H:=\left[c_{1}, c_{1+m}, c_{1+2 m}, \ldots, c_{1+(q-3) m}, \zeta_{m}\right] .
$$

Since $\rho(k m)=\rho(m)>(q-2) m$ for $k=2,3, \ldots, q-3$ by Lemma 4.10, the map $f^{\circ m}$ sends the arc $\left[c_{1}, c_{1+k m}\right]$ homeomorphically onto its image, and the same is obviously true for $\left[c_{1}, \zeta_{m}\right]$. We thus get a homeomorphism $f^{\circ m}: H \rightarrow H^{\prime}$ with

$$
H^{\prime}=\left[c_{1+m}, c_{1+2 m}, c_{1+3 m}, \ldots, c_{1+(q-2) m}, c_{1}\right] .
$$

Since $\zeta_{m} \in\left[c_{1+(q-2) m}, c_{1}\right]$, we have $H \subset H^{\prime} \subset H \cup\left[\zeta_{m}, c_{1+(q-2) m}\right]$. Moreover, $\zeta_{r} \in$ $\left[c_{1+k m}, c_{1+(q-2) m}\right]$ for $k=0,1, \ldots, q-3$ : the first difference between the itineraries of $c_{1+(q-2) m}$ and $c_{1}$ occurs at position $r$, while $c_{1}$ and $c_{1+k m}$ have at least $m$ identical entries. Since $\zeta_{m} \in\left[\zeta_{r}, c_{1}\right]$, it follows similarly that $\zeta_{m} \in\left[\zeta_{r}, c_{1+k m}\right] \subset\left[c_{1+(q-2) m}, c_{1+k m}\right]$ for $k \leq q-3$. Therefore, $H^{\prime} \backslash H=\left(\zeta_{m}, c_{1+(q-2) m}\right]$.

Among the endpoints defining $H$, only $c_{1+(q-3) m}$ maps outside $H$ under $f^{\circ m}$, so $c_{1+(q-3) m}$ is an endpoint of $H$ and thus also of $H^{\prime}$. It follows that $c_{1+(q-4) m}$ is an endpoint of $H$ and thus also of $H^{\prime}$ and so on, so $c_{1}, \ldots, c_{1+(q-2) m}$ are endpoints of $H$. Finally, also $\zeta_{m}$ is an endpoint of $H$ (or $c_{1}$ would be an inner point of $H^{\prime}$ ). As a result, $H$ and $H^{\prime}$ have the same branch points.

If $q=3$, then $H$ is simply an arc which is mapped in an orientation reversing manner over itself, and hence contains a fixed point of $f^{\circ m}$. Otherwise $H$ contains a branch point. Since $f^{\circ m}$ maps $H$ homeomorphically onto $H^{\prime} \supset H$, it permutes the branch points of $H$. By expansivity there can be at most one branch point, say $z_{1}$, which must be fixed under $f^{\circ m}$. Since $f^{\circ m}:\left[z_{1}, c_{1}\right] \rightarrow\left[z_{1}, c_{1+m}\right]$ is a homeomorphism with $\left[z_{1}, c_{1}\right] \cap\left[z_{1}, c_{1+m}\right]=\left\{z_{1}\right\}$, the arc $\left(z_{1}, c_{1}\right]$ cannot contain a point on the orbit of $z_{1}$, so $z_{1}$ is characteristic.

If $z_{1}$ is a tame branch point, then $\zeta_{m} \in\left[z_{1}, 0\right]$ by Lemma 4.9, and $m$ occurs in the internal address in contradiction to the failing admissibility condition. If $z_{1}$ has exactly two arms, these are interchanged by $f^{\circ m}$, and $\zeta_{m} \in G_{0}$, the global arm containing 0 . By Lemma $4.9(2), \zeta_{m} \in\left[0, z_{1}\right]$ and $m$ occurs in the internal address, again a contradiction. Hence $z_{1}$ is an evil branch point. 
Now $H$ has exactly $q-1$ endpoints, and these are contained in different global arms of $z_{1}$. The corresponding local arms are permuted transitively by $f^{\circ m}$. Since $z_{1}$ is evil, it has exactly $q$ arms.

This also concludes the proof of Theorem 4.2.

\subsection{Definition (Upper and Lower Kneading Sequences)}

If $\nu$ is a $\star$-periodic kneading sequence of exact period $n$, we obtain two periodic kneading sequences $\nu_{0}$ and $\nu_{1}$ by consistently replacing every $\star$ with 0 (respectively with 1 ); both sequences are periodic with period $n$ or dividing $n$, and exactly one of them contains the entry $n$ in its internal address. The one which does is called the upper kneading sequence associated to $\nu$ and denoted $\mathcal{A}(\nu)$, and the other one is called the lower kneading sequence associated to $\nu$ and denoted $\overline{\mathcal{A}}(\nu)$.

\subsection{Lemma (Itinerary Immediately Before $c_{1}$ )}

When $x \rightarrow c_{1}$ in a Hubbard tree for the $\star$-periodic kneading sequence $\nu$, the itinerary of $x$ converges (pointwise) to $\overline{\mathcal{A}}(\nu)$.

Proof. Let $\tau$ be the limiting itinerary of $x$ as $x \rightarrow c_{1}$ and let $n$ be the period of $\nu$. Then $\tau$ is clearly periodic with period (dividing) $n$ and contains no $\star$, so $\tau \in$ $\{\mathcal{A}(\nu), \overline{\mathcal{A}}(\nu)\}$. Let $m$ be the largest entry in the internal address of $\nu$ which is less than $n$. Then there is a closest precritical point $\zeta_{m} \in\left[0, c_{1}\right)$ (Lemma 4.7) and $f^{\circ n}$ maps $\left[\zeta_{m}, c_{1}\right]$ homeomorphically onto its image. Since $f^{\circ m}$ sends $\left(\zeta_{m}, c_{1}\right) \ni x$ onto $\left(c_{1}, c_{1+m}\right) \ni f^{\circ m}(x)$, which is sent by $f^{\circ(n-m)}$ onto $\left(c_{1+n-m}, c_{1+n}\right)$, we get $\tau_{1} \ldots \tau_{n-m}=$ $\nu_{1} \ldots \nu_{n-m}=\tau_{m+1} \ldots \tau_{n}$. Hence $\rho_{\tau}(m)>n$; since $m$ occurs in the internal address of $\tau$, the number $n$ does not.

\subsection{Proposition (Exact Period of Kneading Sequence)}

For every $\star$-periodic kneading sequence of period $n$, the associated upper kneading sequence $\mathcal{A}(\nu)$ has exact period $n$.

Proof. Let $\tau:=\mathcal{A}(\nu)$ be the upper kneading sequence associated to $\nu$ and suppose by contradiction that the exact period of $\tau$ is $m<n$. Then $\nu$ fails the admissibility condition for period $m$ : since $\rho_{\tau}(m)=\infty$ and $n$ is in the internal address of $\tau$ by assumption, $m$ cannot occur on the internal address of $\tau$ and hence neither on the internal address of $\nu$. If $\rho_{\tau}(k) \geq m$ for a proper divisor $k$ of $m$, then the exact period of $\tau$ would be less than $m$, a contradiction. Hence $\rho_{\nu}(k)=\rho_{\tau}(k)<m$. The third part of the admissibility condition is clear because $r=m$.

Thus by Theorem 4.2 the Hubbard tree for $\nu$, say $(T, f)$, has an evil orbit with period $m$. Let $z_{1}$ be its characteristic point; it has itinerary $\tau$. Then $f^{\circ n}$ sends $\left[z_{1}, c_{1}\right]$ homeomorphically onto itself, so all points on $\left[z_{1}, c_{1}\right)$ have itinerary $\tau$. By Lemma 4.15, it follows that $\tau$ is the lower kneading sequence associated to $\nu$, a contradiction. 


\subsection{Lemma (Characteristic Points and Upper Sequences)}

Let $z$ be a characteristic point with itinerary $\tau$ and exact period $n$. Then exactly one of the following two cases holds:

(1) - all local arms are permuted transitively, i.e., z is tame,

- the internal address of $\tau$ contains the entry $n$,

- the exact period of $\tau$ equals $n$,

- $\tau=\mathcal{A}(\nu)$ for some $\star$-periodic kneading sequence $\nu$ of exact period $n$.

(2) - the local arm towards 0 is fixed, all others are permuted transitively,

- the internal address of $\tau$ does not contain the entry $n$,

- if the exact period of $z$ and $\tau$ coincide then $\tau=\overline{\mathcal{A}}(\nu)$ for some $\star$-periodic kneading sequence $\nu$ of exact period $n$.

For any $\star$-periodic sequence $\nu$, there is at most one tame periodic point in $T$ such that $\tau(p)=\mathcal{A}(\nu)$.

Proof. By Corollary 3.4 either all local arms at $z$ are permuted transitively or the local arm pointing to 0 is fixed and all others are permuted transitively. By Proposition 3.8, $n$ is contained in the internal address of $\tau$ if and only of the local arm towards 0 is not fixed.

Let $n^{\prime}$ be the exact period of $\tau$. Then $n=k n^{\prime}$ for some $k \geq 1$ and $\rho_{\tau}(n)=\infty$. Therefore, if $n$ is contained in the internal address of $\tau$ then $k=1$ by the last assertion of Lemma 2.5.

The last remaining property of the first case follows immediately from $n=n^{\prime}$, the definition of upper and lower kneading sequences and Proposition 4.16. Similarly, the third property in the second case follows from these results.

For the last statement, let us assume that there are two tame periodic points $p, q$ with itinerary $\mathcal{A}(\nu)$. Then they have both exact period $n^{\prime}$ and $f^{\circ n^{\prime}}([p, q])=[p, q]$. Thus not all local arms of $p, q$ are permuted transitively and neither $p$ nor $q$ is tame, a contradiction.

An immediate corollary of the preceding lemma is that if the period of $z$ and of $\tau$ coincide, then the type of $z$ (tame or not) is completely encoded in $\tau$.

In the second case however, if the exact period of $z$ and $\tau$ do not coincide, then $\tau$ may equal the upper or the lower kneading sequence of some $\star$-periodic kneading $\nu$ of exact period $n^{\prime}$.

\subsection{Lemma (Periodic Point behind Closest Precritical Point)}

Let $\zeta \in\left[0, c_{1}\right)$ be a precritical point with $\operatorname{STEP}(\zeta)=m$ so that $f^{\circ m}:\left[\zeta, c_{1}\right] \rightarrow\left[c_{1}, c_{1+m}\right]$ is homeomorphic. Then the arc $\left(\zeta, c_{1}\right)$ contains a characteristic periodic point $z$ with exact period $m$. The first return map of $z$ fixes no local arm at $z$.

Proof. First we show that $\left(c_{1}, \zeta\right)$ contains a periodic point of period $m$. Assume by contradiction that this is not the case. The construction in BKS2] does not only give 
the existence of the abstract Hubbard tree, but also the existence of extended trees that contain a finite number periodic orbits, see also [BKS1, Theorem 20.12]. Here we will include an $m$-periodic point $p$ with itinerary $\tau=\overline{\nu_{1} \ldots \nu_{m}}$, where $\nu$ is the kneading sequence of the Hubbard tree. If $c_{1}$ is periodic of period $n<m$, then we will use the itinerary $\tilde{\nu}$ of a point $x$ very close to $c_{1}$; so $\tau=\overline{\tilde{\nu}_{1} \ldots \tilde{\nu}_{m}}$. By the choice of $\tau, \zeta$ does not separate $p$ from $c_{1}$. The triod $H_{0}=\left[c_{1}, p, \zeta\right]$ maps under $f^{\circ m}$ homeomorphically onto

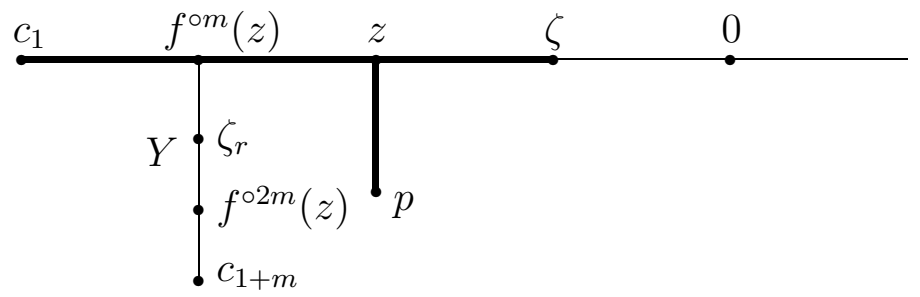

FiguRE 5. Subtree $H_{0}=\left[c_{1}, p, \zeta\right]$ (bold lines) with its image under $f^{\circ m}$.

[ $\left.c_{1+m}, p, c_{1}\right]$, see Figure 5. If $H_{0}$ is degenerate, then it must necessarily have $c_{1}$ in the middle. But then, $f^{\circ m}\left(\left[c_{1}, p, \zeta\right]\right)=\left[c_{1+m}, p, c_{1}\right]$ is degenerate with $c_{1+m}$ in the middle: we have $\zeta \in\left[0, c_{1}\right], c_{1} \in[\zeta, p]$ and $c_{1+m} \in\left[c_{1}, p\right]$, hence $c_{1} \in\left[0, c_{1+m}\right]$ in contradiction to the fact that $c_{1}$ is an endpoint of the Hubbard tree (we cannot have $c_{1+m}=c_{1}$ because then $\zeta=c_{1}$ ).

Hence there is a branch point, say $z$, in the interior of $H_{0}$. Since $z \in(p, \zeta)$, we have $f^{\circ m}(z) \in\left(p, c_{1}\right) \subset[z, p) \cup\left[z, c_{1}\right)$. The possibility $f^{\circ m}(z)=z$ contradicts our initial assumption.

If $f^{\circ m}(z) \in(z, p)$, then $f^{\circ m}$ maps $[z, p]$ homeomorphically into itself, so all points on $[z, p]$ have the same itinerary. This contradicts either expansivity or finiteness of the orbit of the branch point $z$.

Therefore, $f^{\circ m}(z) \in\left(c_{1}, z\right)$. In this case, $f^{\circ m}\left(\left[c_{1}, z\right]\right)$ branches off from $\left[c_{1}, \zeta\right]$ at $f^{\circ m}(z)$; it belongs to an arm $Y$ at $f^{\circ m}(z)$, and $f^{\circ 2 m}(z) \in Y$. By expansivity, $f^{\circ m}$ cannot map $Y$ homeomorphically into itself, so there exists a closest precritical point $\zeta_{k} \in Y$ with $k<m$. By Lemma 4.7, $m \notin \operatorname{orb}_{\rho}(k)$. There is a unique $s \in \operatorname{orb}_{\rho}(k)$ with $s<m<\rho(s)$. Then $f^{\circ m}$ maps the triod $\left[c_{1}, \zeta_{s}, z\right]$ homeomorphically onto the image triod $\left[c_{1+m}, c_{1+m-s}, f^{\circ m}(z)\right]$ with branch point in $\left(f^{\circ m}(z), c_{1+m}\right) \subset Y$. Therefore, $c_{1+m-s} \in Y$. Now let $\zeta^{\prime}$ be the earliest precritical point on $\left[c_{1}, c_{1+m-s}\right]$. By Lemma 4.7, $\operatorname{STEP}\left(\zeta^{\prime}\right)=\rho(m-s)-(m-s)$. The first assertion of Lemma 2.5 states that $m \in$ $\operatorname{orb}_{\rho}(\rho(m-s)-(m-s))$, so $\zeta_{m} \in\left[c_{1}, c_{1+m-s}\right]$. Therefore, $\zeta_{m} \neq \zeta$ (the points $\zeta$ and $\zeta_{m}$ are in different arms at $\left.f^{\circ m}\right)$, and this is a contradiction.

We have now proved the existence of a periodic point $z \in\left(c_{1}, \zeta\right)$ with itinerary $\tau$ and period $m$. It is characteristic: if not, let $z_{1} \in\left(z, c_{1}\right)$ be the characteristic point; then $f^{\circ m}\left(z_{1}, \zeta\right)=\left(z_{1}, c_{1}\right)$ and $z \in\left(z_{1}, \zeta\right)$, which is a contradiction. 
Let $k \mid m$ be the exact period of $z$. By Lemma 3.6, $f^{\circ k}$ sends the local arm at $z$ to 0 either to itself or to the local arm to $c_{1}$. The first case is excluded by the fact that $f^{\circ m}:[z, \zeta] \rightarrow\left[z, c_{1}\right]$ is a homeomorphism. In the second case, $f^{\circ k}:[z, \zeta] \rightarrow\left[z, f^{\circ k}(\zeta)\right] \subset$ $\left[z, c_{1}\right]$ is a homeomorphism. If $k<m$, then $f^{\circ k}(\zeta) \in\left(z, c_{1}\right)$ and $f^{\circ m}$ could not be a homeomorphism on $\left[\zeta, c_{1}\right]$. Hence $k=m$ is the exact period of $z$, and no local arm at $z$ is fixed by $f^{\circ m}$.

For any $m \geq 1$, let $r \in\{1,2, \ldots, m\}$ be congruent to $\rho(m)$ modulo $m$, and define

$$
q(m):= \begin{cases}\frac{\rho(m)-r}{m}+1 & \text { if } m \in \operatorname{orb}_{\rho}(r) \\ \frac{\rho(m)-r}{m}+2 & \text { if } m \notin \operatorname{orb}_{\rho}(r) .\end{cases}
$$

\subsection{Proposition (Number of Arms at Tame Branch Points)}

If $z_{1}$ is a tame branch point of exact period $m$, then $m$ occurs in the internal address, and the number of arms is $q(m)$. Conversely, for any entry $m$ in the internal address with $q(m) \geq 3$, there is a tame branch point of exact period $m$ with $q(m)$ arms (unless the critical orbit has period $m$ ).

PROOF. Let $z_{1}$ be the characteristic point of an orbit of tame branch points with exact period $m$, and let $q^{\prime} \geq 3$ be the number of arms at $z_{1}$. By Lemma 3.6, the critical value cannot be periodic with period less than $m$. By Lemma 4.9 (3) and (1), $m$ occurs in the internal address, $\zeta_{m} \in\left(0, z_{1}\right) \subset G_{0}$, and $\zeta_{\rho(m)} \in\left[z_{1}, c_{1}\right]$. Let $r^{\prime}:=\rho(m)-\left(q^{\prime}-2\right) m$. By Lemma 4.11, $0<r^{\prime} \leq 2 m$. Therefore, by Lemma 4.10, $\rho\left(\left(q^{\prime}-2\right) m\right)=\rho(m)$, so by Lemma 4.8, $f^{\circ\left(q^{\prime}-2\right) m}\left(\zeta_{\rho(m)}\right)=\zeta_{r^{\prime}}$ is a closest precritical point and by Lemma [3.6, $\zeta_{r^{\prime}}=f^{\circ\left(q^{\prime}-2\right) m}\left(\zeta_{\rho(m)}\right) \in G_{q^{\prime}-1}$. Since $\zeta_{m} \in G_{0}$, we have $\zeta_{m} \notin\left[c_{1}, \zeta_{r^{\prime}}\right]$ and thus $m \notin \operatorname{orb}_{\rho}\left(r^{\prime}\right)$ (Lemma 4.7).

If $r^{\prime} \leq m$, then $r=r^{\prime}$ and we are in the case $q(m)=\frac{\rho(m)-r}{m}+2=\frac{\rho(m)-r^{\prime}}{m}+2=q^{\prime}$.

If $r^{\prime}>m$, then $r=r^{\prime}-m$ and $f^{\circ m}\left(\zeta_{r^{\prime}}\right)=\zeta_{r}$ by Lemma 4.8. Then $f^{\circ m}$ maps $\left[z_{1}, \zeta_{r^{\prime}}\right]$ homeomorphically onto $\left[z_{1}, \zeta_{r}\right]$, hence $\zeta_{r} \in G_{0}$. By Lemma 4.9 (2) we find that either $r=m$ or $r<m$ and $\zeta_{m} \in\left[z_{1}, \zeta_{r}\right]$, so in both cases $m \in \operatorname{orb}_{\rho}(r)$. Therefore, $q(m)=\frac{\rho(m)-r}{m}+1=q^{\prime}$. Again $q(m)=q^{\prime}$.

For the converse, let $m$ be an entry in the internal address. By Lemma 4.7, the closest precritical point $\zeta_{m}$ exists on $\left[0, c_{1}\right]$. By Lemma 4.18, $\zeta_{m}$ gives rise to a characteristic point $z_{1} \in\left[\zeta_{m}, c_{1}\right]$ of exact period $m$ and the first return map of $z_{1}$ fixes no local arm. By Lemma $4.9(1), \zeta_{\rho(m)} \in\left[z_{1}, c_{1}\right]$.

If $z_{1}$ is a branch point, then no local arm of $z_{1}$ is fixed by $f^{\circ m}$, so $z_{1}$ is tame. By the first assertion of the lemma, the number of arms is $q(m)$.

Finally, suppose that $z_{1}$ has only two arms $G_{0} \ni 0$ and $G_{1} \ni c_{1}$. If the critical orbit is periodic and $m$ is an entry in the internal address, the period of the critical orbit is at least $m$, and equality is excluded by hypothesis. By Lemma 4.11, we have $\rho(m) \leq 2 m$ and $r^{\prime}:=\rho(m)-m=r$. Then $f^{\circ m}\left(\zeta_{\rho(m)}\right)=\zeta_{r^{\prime}} \in G_{0}$. Lemma 4.9 (2) then gives that $\zeta_{m} \in\left[\zeta_{r}, z_{1}\right]$ and hence $m \in \operatorname{orb}_{\rho}(r)$. It follows that $q(m)=\frac{\rho(m)-r}{m}+1=2$. 
Together, Propositions 4.13 and 4.19 describe all branch points in all Hubbard trees.

\subsection{Corollary (Uniqueness of Hubbard Tree)}

If $(T, f)$ is a Hubbard tree with $\star$-periodic or preperiodic kneading sequence $\nu$, then $\nu$ alone determines $(T, f)$ uniquely up to equivalence.

Proof. By Propositions 4.12 and 4.13 , the tree $(T, f)$ has an evil periodic orbit of exact period $m$ if and only if $\nu$ fails the admissibility condition for period $m$; the number of arms is determined by Proposition 4.13. By Proposition 4.19, there is a branch point of period $m$ only if $m$ occurs in the internal address associated $\nu$; for every $m$ on this internal address, the quantity $q(m)$ from (1) determines whether or not there is a branch point and, if so the number of arms. Every branch point of any period $m$ has the property that the itinerary of the associated characteristic point coincides with $\nu$ for at least $m$ entries; this determines the itinerary of all points on the orbit of every branch point. Finally, every endpoint of $(T, f)$ is on the critical orbit by definition, so the itineraries of endpoints are shifts of $\nu$.

If $\left(T^{\prime}, f^{\prime}\right)$ is another Hubbard tree with kneading sequence $\nu$, then we show that it is equivalent to $(T, f)$ in the sense as defined after Definition 2.2, Itineraries define a bijection between branch points of $(T, f)$ and $\left(T^{\prime}, f^{\prime}\right)$ and between postcritical points, and this bijection is respected by the dynamics. It thus suffices to prove that both trees have the same endpoints and their edges connect corresponding points. Recall that postcritical points and branch points are jointly known as marked points.

To see this, we use precritical points: by definition, these are points $\zeta \in T$ with $f^{\circ k}(\zeta)=c_{0}$ for some $k \geq 0$; in this case we write $\operatorname{STEP}(\zeta)=k$. Every such precritical point $\zeta$ has itinerary $\tau_{1} \tau_{2} \ldots \tau_{k-1} \star \nu$ with $\tau_{1}, \ldots, \tau_{k-1} \in\{0,1\}$. By induction on $k$, we show that such a point $\zeta$ with itinerary $\tau_{1} \tau_{2} \ldots \tau_{k-1} \star \nu$ exists in $T$ if and only if it exists in $T^{\prime}$, and if it does, corresponding marked points are in corresponding components of $T \backslash\{\zeta\}$ resp. $T^{\prime} \backslash\{\zeta\}$. This is obvious for $k=0$ and $\zeta=c_{0}$.

A precritical point $\zeta$ with $\operatorname{STEP}(\zeta)=k+1$ (described by the first $k$ entries $\tau_{0}, \ldots, \tau_{k}$ of its itinerary) obviously exists if and only if there are two postcritical points $x, y \in T$ with $\zeta \in[x, y]$. This is equivalent to the existence of two points $x^{\prime}, y^{\prime} \in T$ which are either postcritical points or precritical points with $\operatorname{STEP}(x) \leq k, \operatorname{STEP}(y) \leq k$ so that $\zeta \in\left[x^{\prime}, y^{\prime}\right]$ and so that $\left(x^{\prime}, y^{\prime}\right)$ does not contain a precritical point $\zeta^{\prime}$ with $\operatorname{STEP}\left(\zeta^{\prime}\right)<k+1$. The latter condition can be checked using the itineraries of $x^{\prime}$ and $y^{\prime}$, and their existence is the same for $T$ and for $T^{\prime}$ by inductive hypothesis.

It now follows easily that $T$ and $T^{\prime}$ have endpoints with identical itineraries, so they have a natural bijection between marked points. It also follows that every precritical point $\zeta$ disconnects $T$ and $T^{\prime}$ into two parts so that corresponding parts contain marked points with identical itineraries, and this implies that the edges of $T$ and $T^{\prime}$ connect corresponding points. This means by definition that $(T, f)$ and $\left(T^{\prime}, f^{\prime}\right)$ are equivalent as claimed. 


\section{REFERENCES}

[BFH] Ben Bielefeld, Yuval Fisher, John Hubbard: The classification of critically preperiodic polynomials as dynamical systems, Journal AMS 54 (1992) 721-762.

[BKS1] Henk Bruin, Alexandra Kaffl, Dierk Schleicher, Symbolic dynamics of quadratic polynomials, Monograph, in preparation. A preliminary version (Preprint 2002) is available on the webpages of the Mittag-Leffler institute: http://www.ml.kva.se/preprints/archive2001-2002.php

[BKS2] Henk Bruin, Alexandra Kaffl and Dierk Schleicher, Existence of quadratic Hubbard trees, Preprint 2007.

[DH1] Adrien Douady, John Hubbard, Études dynamique des polynômes complexes I \&3 II, Publ. Math. Orsay. (1984-85) (The Orsay notes).

[DH2] Adrien Douady, John Hubbard, A proof of Thurston's topological characterization of rational functions, Acta Mathematica 171 (1993) 263-297.

[D] Adrien Douady, Julia sets and the Mandelbrot set, in: H.-O. Peitgen, P. Richter: The beauty of fractals, Springer-Verlag, New York (1986) 161-173.

[HS] John Hubbard, Dierk Schleicher, The spider algorithm, Proc. of Symp. Appl. Math. 49 Complex dynamical systems: the mathematics behind the Mandelbrot and Julia sets (1994) 155-180.

[K] Alexandra Kaffl, On the structure of abstract Hubbard trees and the space of abstract kneading sequences of degree two, Ergod. Thy. Dyn. Sys 27 (2007), 1215-1238.

[Ka] Virpi Kauko, Trees of visible components in the Mandelbrot set, Fund. Math. 164 (2000) 41-60.

[Ke] Karsten Keller, Invariant factors, Julia equivalences and the (abstract) Mandelbrot set, Springer Lect. Notes Math. 1732 (2000).

[LS] Eike Lau, Dierk Schleicher, Internal addresses in the Mandelbrot set and irreducibility of polynomials, Stony Brook Preprint \#19 (1994).

[M] John Milnor, Dynamics in one complex variable. Introductory lectures, Friedr. Vieweg \& Sohn, Braunschweig (1999).

[MT] John Milnor, William Thurston, On iterated maps of the interval, LNM 1342 (J. C. Alexander ed.). 465-563 (1988).

[Pe] Chris Penrose, On quotients of shifts associated with dendrite Julia sets of quadratic polynomials, Thesis, University of Coventry, (1994).

[Po] Alfredo Poirier, On postcritically finite polynomials, part two: Hubbard trees, Stony Brook Preprint \#17 (1993).

[S1] Dierk Schleicher, Internal addresses in the Mandelbrot set and irreducibility of polynomials. Submitted (2007). ArXiv math.DS/9411238.

[S2] Dierk Schleicher, Rational parameter rays of the Mandelbrot set. Asterisque 261 (2000), 409447.

[S3] Dierk Schleicher, On Fibers and Local Connectivity of Mandelbrot and Multibrot Sets. In: Lapidus, Frankenhuysen (eds), a Mandelbrot Jubilee. Proc Symp Pure Math 72 (2004), 477507

Department of Mathematics, University of Surrey, Guildford GU2 7XH, United KINGDOM

E-mail address: H.Bruin@surrey.ac.uk

School of Engineering and Science, Jacobs University Bremen, P.O. Box 750561 , D-28725 BRemen, Germany

E-mail address: dierk@jacobs-university.de 